\title{
Retraction Note to: Genome Shuffling Enhances Lipase Production of Thermophilic Geobacillus Sp
}

\author{
Pornchanok Chalopagorn ${ }^{1}$. \\ Jittima Charoenpanich ${ }^{2,3,4}$. \\ Kiattawee Choowongkomon ${ }^{1,5}$
}

Published online: 3 June 2017

(C) Springer Science+Business Media New York 2017

\section{Retraction to: Appl Biochem Biotechnol (2014) 174:1444-1454 DOI 10.1007/s12010-014-1109-6}

Per the request of the Faculty of Science, Kasetsart University, who conducted an internal investigation, this article has been retracted due a conflict concerning the origin of science discussed in this article.

Jittima Charoenpanich

jittima@buu.ac.th

$\square$ Kiattawee Choowongkomon

fsciktc@ku.ac.th

1 Department of Biochemistry, Faculty of Science, Kasetsart University, 50 Ngam Wong Wan Rd, Chatuchak, Bangkok 10900, Thailand

2 Department of Biochemistry and Centre of Excellence for Innovation in Chemistry (PERCH-CIC), Faculty of Science, Burapha University, Bangsaen, Chonburi 20131, Thailand

3 Environmental Science Program, Faculty of Science, Burapha University, Bangsaen, Chonburi 20131, Thailand

4 Center of Excellence on Environmental Health and Toxicology (CHE), Ministry of Education, Bangkok, Thailand

5 Center for Advanced Studies in Tropical Natural Resources, National Research University-Kasetsart University, Thailand CAST?NAR, Bangkok, NRU- KU, Thailand 